\title{
Dynamic financial planning: certainty equivalents, stochastic constraints and functional conjugate duality
}

\section{Thomas R. Jefferson \& Carlton H. Scott}

To cite this article: Thomas R. Jefferson \& Carlton H. Scott (2005) Dynamic financial planning: certainty equivalents, stochastic constraints and functional conjugate duality, IIE Transactions, $37: 10,931-938$

To link to this article: http://dx.doi.org/10.1080/07408170591007830

曲 Published online: 23 Feb 2007.

Submit your article to this journal $\widetilde{ }$

Џ Article views: 26

Q View related articles $\sqsubset$

4 Citing articles: 2 View citing articles 


\title{
Dynamic financial planning: certainty equivalents, stochastic constraints and functional conjugate duality
}

\author{
THOMAS R. JEFFERSON ${ }^{1}$ and CARLTON H. SCOTT ${ }^{2}$ \\ ${ }^{1}$ Faculty of Business Administration, Bilkent University, Bilkent 06800, Ankara, Turkey \\ E-mail: jeffers@bilkent.edu.tr \\ ${ }^{2}$ Graduate School of Management, University of California, Irvine, CA 92717, USA \\ E-mail: chscott@uci.edu
}

\begin{abstract}
This paper studies portfolios under risk and stochastic constraints. Certainty equivalents combine risk aversion and exponential utility to form the objective. Budget and stochastic constraints on the account balance are used to ensure a positive net worth over time. These portfolio models are analyzed by functional conjugate duality for general distributions and by conjugate duality for the normal distribution. All the programs are convex. The duals provide insight into this approach and relate it to other stochastic and financial concepts.
\end{abstract}

\section{Introduction}

One of the major thrusts in financial engineering is the management and optimization of risky portfolios. Although this is a well-researched area (Merton, 1990), it is still a mine of unsolved and intractable problems that has attracted the attention of researchers from a diversity of disciplines including economics, mathematics, computer science, physics and engineering. The basic portfolio problem involves the allocation of a fixed amount of wealth among a finite set of investment alternatives where each alternative has an uncertain return. In one of the earliest models (Markowitz, 1952), the objective is to maximize the expected return while minimizing risk as measured by the variance-covariance matrix of the portfolio. Current research generally focuses on an objective of the investor's expected lifetime utility and attempts to incorporate issues such as taxes and investor preferences as well as risk (Ziemba and Mulvey, 1998). Another approach focuses on shorter term percentile measures such as Value at Risk (VaR) or Conditional Value at Risk (CVaR) (Jorion, 2000; Uryasev, 2000). Solution procedures are generally based on stochastic dynamic programming or stochastic programming (Kall and Wallace, 1994; Prekopa, 1995; Birge and Louveaux, 1997). One approach to risk that has had significant success in management applications is to treat uncertainty by a certainty equivalent (Pratt, 1964). This is the approach that we adopt in this paper.

In an earlier paper, the risky static-portfolio selection problem was embedded into function space and analyzed by certainty equivalents and geometric programming for functionals (Jefferson et al., 1979). In this paper, we first revisit the static model before going on to study the risky dynamic portfolio problem including stochastic constraints with a view to providing insight on this important class of problems through analysis and duality.

The first feature of the risky portfolio problem is the use of certainty equivalents to reduce risk. Instead of maximizing return, Pratt (1964) proposed that the utility be maximized under the assumption that the decision-maker has an exponential utility function: $U(x)=1 / r(1-\exp (-r x))$. If $p$ is the random variable of return, then the certainty equivalent, $\pi$, is proven by Pratt to be independent of the initial wealth: $\pi(p)=-1 / r \ln \left(E_{p}[\exp (-r p)]\right)$, where $r>0$, is the risk aversion parameter for the decision-maker. In the limit, as $r$ goes to zero, the certainty equivalent function $\pi$ tends to the risk-neutral function of the return. Thus, in the risky portfolio problem, we will want to maximize the present value of the certainty equivalents over a given time horizon.

\section{The models}

\subsection{The static model}

The foundation of the risky dynamic portfolio problem is the risky static-portfolio problem. Suppose there is a budget of $C$ and $n$ projects, each with a cost of $C_{j}$ and a stochastic return of $x_{j}, j=1, \ldots, n$. Let $s_{j}$ be the fraction of the $j$ th 
project invested in, then the formulation is:

$$
\max -\sum_{j=1}^{n}\left(1 / r \ln \left(E\left[\exp \left(-r s_{j} x_{j}\right)\right]\right)+s_{j} C_{j}\right),
$$

subject to

$$
\begin{aligned}
& \sum_{j=1}^{n} s_{j} C_{j} \leq C, \\
& 0 \leq s_{j} \leq 1, \forall j .
\end{aligned}
$$

In Jefferson et al. (1977), the static model was optimized over the $s_{j}$. Here, to gain insight into the risk-aversion parameter $r$, the model will be optimized over $r$ and $s_{j}$. Further insights are obtained when the random variables are assumed to be independent normal. Both of these models with be analyzed using the duality presented in the next section

\subsection{The dynamic model}

A more realistic, but more complicated portfolio model has returns or costs spread over a time horizon and an objective of maximizing the risk-adjusted discounted cash flow. Index the present and future cash flows over $t=0, \ldots, T$ and let the interest rate be $i$. The risk-adjusted dynamic portfolio model is:

$\max -\sum_{t=0}^{T}(1+i)^{-t} \sum_{j=1}^{n}\left(1 / r \ln E\left[\exp \left(-r s_{j} x_{t j}\right)\right]\right)-\sum_{j=1}^{n} s_{j} C_{j}$,

subject to

$$
\begin{aligned}
\sum_{j=1}^{n} s_{j} C_{j} & \leq C, \\
0 & \leq s_{j} \leq 1, \forall j .
\end{aligned}
$$

Typically the portfolio will be reviewed yearly or more frequently and updated with new figures, information and projects. It is the adaptive use of the portfolio model, which makes it truly dynamic. Again, to gain insight into the dynamic portfolio model, it will be analyzed for general distributions and for a typical normal distribution using duality.

\subsection{The dynamic model with stochastic constraints}

Whereas certainty equivalents lead to a conservative portfolio, they do not check each time period to see that we are running a positive balance; however, our bankers will do so. In order to ensure that we run a positive balance in every time period, stochastic constraints are required. This leads to the third model under consideration. Here the probably of the accrued cash balance being negative is kept to a small probability $p_{T^{\prime}}, 0 \leq T^{\prime} \leq T$ :

$$
\begin{aligned}
& \max -\sum_{t=0}^{T}(1+i)^{-t} \sum_{j=1}^{n}\left(1 / r \ln \left(E\left[\exp \left(-r s_{j} x_{t j}\right)\right]\right)\right)- \\
& \sum_{j=1}^{n} s_{j} C_{j}
\end{aligned}
$$

subject to

$$
\begin{aligned}
& \int \mathbf{1}_{N}\left[\sum_{t=0}^{T^{\prime}}(1+i)^{T^{\prime}-t} \sum_{j=1}^{n} s_{j} x_{t j}+(1+i)^{T^{\prime}}\left(C-\sum_{j^{\prime}=1}^{n} s_{j^{\prime}} C_{j^{\prime}}\right)\right] \\
& \quad \times \mathrm{d} F(x) \leq p_{T^{\prime}}, \quad 0 \leq T^{\prime} \leq T \\
& \sum_{j=1}^{n} s_{j} C_{j} \leq C, \\
& 0 \leq s_{j} \leq 1, \quad \forall j .
\end{aligned}
$$

The indicator function $\mathbf{1}_{N}()$ in stochastic constraint (8), is one when its argument is negative and zero elsewhere.

The next section contains the duality theory for convex functionals needed to analyze the portfolio models presented above. This takes place in Section 4 of the paper. Results and conclusions are summarized in the final section.

\section{Conjugate duality for convex functionals}

In order to understand the models for dynamic financial planning we will call on a variety of conjugate duals. The most general is generalized geometric programming for functionals developed by Scott and Jefferson (1977). Geometric programming over functionals was also derived in the same paper. When the probability density functions are known, the formulations can be analyzed by generalized geometric programming (Peterson, 1976) and in some cases by geometric programming (Duffin et al., 1967). For functionals, consider the space of mappings of the random portfolio returns $z(x): X \rightarrow R$. We assume that this space is a measurable, complete, separable, real metric space with inner product: $\left\langle z, z^{*}\right\rangle=\int z(x) z^{*}(x) \mathrm{d} x$. Let $G(z(x))$ be a convex functional then the following properties of convex functionals over convex sets can be defined in preparation for calculation of the dual program.

Definition 1. The subgradient set of a convex functional $G$ defined over a convex domain $\Gamma$ at a point $x_{0}$ is defined by:

$\partial G\left(z_{0}(x)\right)=\left\{z^{*} \mid G(z(x))-\left\langle z^{*}, z-z_{0}\right\rangle \leq G\left(z_{0}(x)\right), \forall z \in \Gamma\right\}$.

The subgradient set is the set of all sublinear approximations of $G$ and is the basis for duality.

Definition 2. The conjugate transform of a convex functional $G$ defined on the convex domain $\Gamma$ is $G^{*}$ defined on 
$\Gamma^{*}$ where:

$$
G^{*}\left(z^{*}\right)=\sup _{z \in \Gamma}\left\{\left\langle z, z^{*}\right\rangle-G(z)\right\},
$$

and

$$
\Gamma^{*}=\left\{z^{*} \mid\left\langle z, z^{*}\right\rangle-G(z)<\infty\right\} .
$$

The conjugate inequality is a consequence of the definition of the conjugate transform: $G(z)+G^{*}\left(z^{*}\right) \geq$ $\left\langle z, z^{*}\right\rangle$ with the supremum of the conjugate transform being achieved and the conjugate inequality equal when $z^{*} \in \partial G(z)$ provided $G$ is a closed convex functional.

Definition 3. The positive homogeneous extension of a convex functional $G$ on convex domain $\Gamma$ is:

$$
\begin{aligned}
G^{+}(z, \lambda)= & \begin{cases}\lambda G(z / \lambda), & \lambda>0, \\
\sup _{z^{*} \in \Gamma^{*}}\left\langle z, z^{*}\right\rangle, & \lambda=0,\end{cases} \\
\Gamma^{+}= & \left\{(z, \lambda) \mid \sup _{z^{*} \in \Gamma^{*}}\left\langle z, z^{*}\right\rangle<\infty, \lambda=0\right\} \\
& \times \cup\{(z, \lambda) \mid z / \lambda \in \Gamma, \lambda>0\} .
\end{aligned}
$$

The positive homogeneous extension is important because the conjugate transform of the 0 functional on the set $\{z \mid G(z) \leq 0$ and $z \in \Gamma\}$ is $G^{*+}\left(z^{*}, \lambda^{*}\right)$ defined on the convex set $\Gamma^{*+}$.

Consider the following convex program:

Program A:

$$
\inf G_{0}\left(z_{0}\right)+G_{1}^{+}\left(z_{1}, \lambda\right)
$$

subject to

$$
\begin{aligned}
G_{2}\left(z_{2}\right) & \leq 0, \\
z_{0} & \in \Gamma_{0},\left(z_{1}, \lambda\right) \in \Gamma_{1}^{+}, z_{2} \in \Gamma_{2}, \\
z & =\left(z_{0}, z_{1}, z_{2}\right) \in \chi,
\end{aligned}
$$

where $\chi$ is a subspace.

Using the definitions above the conjugate dual to program A can be derived and is:

\section{Program B:}

$$
\inf G_{0}^{*}\left(z_{0}^{*}\right)+G_{2}^{*+}\left(z_{2}^{*}, \mu^{*}\right),
$$

subject to

$$
\begin{aligned}
G_{1}^{*}\left(z_{1}^{*}\right) & \leq 0, \\
z_{0}^{*} & \in \Gamma_{0}^{*}, z_{1}^{*} \in \Gamma_{1}^{*},\left(z_{2}^{*}, \mu^{*}\right) \in \Gamma_{2}^{*+}, \\
z^{*} & =\left(z_{0}^{*}, z_{1}^{*}, z_{2}^{*}\right) \in \chi^{\perp},
\end{aligned}
$$

where $\chi^{\perp}$ is the orthogonal complementary subspace to $\chi$.

Note that there can be a multiplicity of constraints in either program A or program B. Scott and Jefferson (1977) prove that provided both Programs A and B are consistent the sum of the objective functions for feasible $x$ and $x^{*}$ is non-negative and optimal solutions are related by the following three conditions:

$$
\begin{aligned}
& \text { 1. } G_{0}\left(z_{0}\right)+G_{1}^{+}\left(z_{1}, \lambda\right)+G_{0}^{*}\left(z_{0}^{*}\right)+G_{2}^{*+}\left(z_{2}^{*}, \mu^{*}\right)=0, \\
& \text { 2. }\left\langle z, z^{*}\right\rangle=0, \\
& \text { 3. } z_{0}^{*} \in \partial G_{0}\left(z_{0}\right) \quad \text { or } \quad z_{0} \in \partial G_{0}^{*}\left(z_{0}^{*}\right), \\
& \quad z_{1}^{*} \in \partial G_{1}\left(z_{1} / \lambda\right) \quad \text { or } \quad z_{1} / \lambda \in \partial G_{1}^{*}\left(z_{1}^{*}\right), \lambda>0, \\
& z_{2}^{*} / \mu^{*} \in \partial G_{2}\left(z_{2}\right) \text { or } z_{2} \in \partial G_{2}^{*}\left(z_{2}^{*} / \mu^{*}\right), \mu^{*}>0 .
\end{aligned}
$$

The three optimality conditions allow the solution of one program from the other. These together with both programs can offer insights into the problem and its solution.

\section{Analysis of stochastic dynamic portfolios}

We return now to the three portfolio problems presented in the Introduction and analyze them using conjugate duality.

\subsection{The static model}

The static portfolio has already been studied in Jefferson et al. (1979) but there is more that can be revealed if we ask what is a good value for the risk-aversion parameter $r$ ? It is reasonable to consider that the budget constraint is binding as one could simply add a project, which is to put the funds into a government bond. Thus, cost can be dropped from the objective, as it is a constant. Finally modify the certainty equivalent by subtracting the reciprocal of $r$, as it is reasonable to assume that the risk would be low for high wealth and high for low wealth. Define $\lambda$ so that $r=1 / \lambda$ and $\lambda \propto C$. This modified certainty equivalent has meaning, as it has a dual relationship with entropy in general and with portfolio variance in particular as will be seen in the analysis. Then the static model can be written as:

$$
\max -\sum_{j=1}^{n}\left(\lambda \ln \left(E\left[\exp \left(z_{j}\left(x_{j}\right) / \lambda\right)\right]\right)\right)-\lambda,
$$

subject to

$$
\begin{aligned}
z_{j}\left(x_{j}\right) & =-s_{j} x_{j}, \quad \forall j, \\
\sum_{j=1}^{n} s_{j} C_{j} & \leq C, \\
0 & \leq s_{j} \leq 1, \quad \forall j, \quad \lambda \geq 0
\end{aligned}
$$

Let $f_{j}\left(x_{j}\right)$ be the marginal probability density function for the probability distribution of $x_{j}$ and converting the objective into an equivalent minimization problem we see that the objective takes the form of $G_{1}^{+}$. The formulation 
then is:

$$
\min \sum_{j=1}^{n}\left(\lambda \ln \int \exp \left(z_{j}\left(x_{j}\right) / \lambda\right) f_{j}\left(x_{j}\right) \mathrm{d} x_{j}\right)+\lambda,
$$

subject to

$$
\begin{aligned}
z_{j}\left(x_{j}\right) & =-s_{j} x_{j}, \quad \forall j, \\
\sum_{j=1}^{n} s_{j} C_{j} & \leq C, \\
0 \leq s_{j} & \leq 1, \quad \forall j, \quad \lambda \geq 0 .
\end{aligned}
$$

Dual program:

$$
\min \sum_{j=1}^{n} \alpha_{j}^{*}+C \gamma^{*}
$$

subject to

$$
\begin{gathered}
\sum_{j=1}^{n} \int z_{j}^{*}\left(x_{j}\right) \ln \left(z_{j}^{*}\left(x_{j}\right) / f_{j}\left(x_{j}\right)\right) \mathrm{d} x_{j} \leq 1 . \\
\int z_{j}^{*}\left(x_{j}\right) \mathrm{d} x_{j}=1, z_{j}^{*}\left(x_{j}\right) \geq 0, \\
\int x_{j} z_{j}^{*}\left(x_{j}\right) \mathrm{d} x_{j} \leq C_{j}\left(1+\gamma^{*}\right)+\alpha_{j}^{*}, \\
\gamma^{*} \geq 0, \alpha_{j}^{*} \geq 0, \forall j .
\end{gathered}
$$

At optimality:

$$
\begin{aligned}
&-\sum_{j=1}^{n}(\lambda \ln (E {\left.\left[\exp \left(z_{j}\left(x_{j}\right) / \lambda\right)\right]\right)-\lambda } \\
&= \sum_{j=1}^{n} \alpha_{j}^{*}+C \gamma^{*}, \\
& z_{j}^{*}\left(x_{j}\right)=\frac{f_{j}\left(x_{j}\right) \exp \left(-s_{j} x_{j}\right)}{\int f_{j}\left(x_{j}\right) \exp \left(-s_{j} x_{j}\right) \mathrm{d} x_{j}}, \quad \forall j,
\end{aligned}
$$

where $\gamma^{*}$ is the dual variable of the budget constraint of Equation (33) and is positive. The $\alpha_{j}^{*}$ are the dual variables for the fraction of the project being invested in Equation (34). It may be zero or positive, depending on the constraint being binding. The sum of these variables, with $\gamma^{*}$ multiplied by the budget value, $C$, is the dual objective and is equal to the original objective. The optimality condition of Equation (40) says that the dual variable $z_{j}^{*}$ is a probability distribution discounted by the investment. Note that the maximization of the certainty equivalent profit implies that discounted entropy is constrained. Fang et al. (1997) study entropy optimization. Furthermore, the reciprocal of the dual variable of the entropy constraint of Equation (36), $\lambda$, is the optimal risk-aversion parameter $r$.

Up to this point the results do not require any assumption on the distribution of the returns. If the returns on the portfolios are assumed to be independent normal, $N\left(\mu_{j}, \sigma_{j}^{2}\right)$, then the portfolio problem becomes simpler. Note that the moment generating function for the normal distribution is:

$$
M_{j}\left(-r s_{j}\right)=\exp \left(-r s_{j} \mu_{j}+r^{2} s_{j}^{2} \sigma_{j}^{2} / 2\right),
$$

and thus

$$
\pi\left(s_{j} x_{j}\right)=-\frac{1}{r} \ln \left(M_{j}\left(-r s_{j}\right)\right)
$$

\subsubsection{The Normal case}

$$
\min \sum_{j=1}^{n}\left(s_{j}^{2} \sigma_{j}^{2} / 2 \lambda\right)-\sum_{j=1}^{n} s_{j} \mu_{j}+\lambda,
$$

subject to

$$
\begin{aligned}
\sum_{j=1}^{n} s_{j} C_{j} & \leq C, \\
0 & \leq s_{j} \leq 1, \quad \forall j, \quad \lambda \geq 0 .
\end{aligned}
$$

Dual program:

$$
\min \sum_{j=1}^{n} \alpha_{j}^{*}+C \gamma^{*}
$$

subject to

$$
\begin{aligned}
& \sum_{j=1}^{n} \frac{\left(s_{j}^{*}+\mu_{j}\right)^{2}}{2 \sigma_{j}^{2}} \leq 1, \\
& C_{j} \gamma^{*}+\alpha_{j}^{*}+s_{j}^{*} \geq 0, \quad \forall j, \\
& \gamma^{*} \geq 0, \quad \alpha_{j}^{*} \geq 0, \quad \forall j .
\end{aligned}
$$

At optimality:

$$
s_{j} / \lambda=\left(s_{j}^{*}+\mu_{j}\right) / \sigma_{j}^{2}, \quad \forall j .
$$

Substituting into the dual nonlinear constraint gives the following constraint:

$$
\sum_{j=1}^{n} \sigma_{j}^{2} s_{j}^{2} /\left(2 \lambda^{2}\right) \leq 1 .
$$

This means that the optimal value of $\lambda^{2}$ is half the variance of the portfolio. Thus, $r$ is $\sqrt{2}$ divided by the standard deviation of the portfolio. This provides a link between risk and variance. The original paper on portfolio analysis by Markowitz (1952) uses a similar objective and his parameter to balance return and risk is equivalent to $r$ or $\lambda$. This is also shown to be equivalent to $\mathrm{CVaR}$ or $\mathrm{VaR}$ in this case (Rockafellar and Uryasev, 2000).

\subsection{The dynamic model}

Let us now fix the risk aversion parameter $r$ and look at the dynamic model. The risk-adjusted dynamic portfolio program is equivalent to:

$$
\min \sum_{t=0}^{T} \frac{(1+i)^{-t}}{r} \sum_{j=1}^{n}\left(\ln \left(E\left[\exp \left(-r s_{j} x_{t j}\right)\right]\right)+\sum_{j=1}^{n} s_{j} C_{j},\right.
$$


subject to

$$
\begin{aligned}
\sum_{j=1}^{n} s_{j} C_{j} & \leq C, \\
0 & \leq s_{j} \leq 1, \quad \forall j .
\end{aligned}
$$

Dual program:

$$
\begin{aligned}
\min & \sum_{t=0}^{T} \sum_{j=1}^{n} \int z_{t j}^{*}\left(x_{t j}\right) \ln \left[z_{t j}^{*}\left(x_{t j}\right) r(1+i)^{t} / f_{t j}\left(x_{t j}\right)\right] \mathrm{d} x_{t j} \\
& +\sum_{j=1}^{n} \alpha_{j}^{*}+C \gamma^{*}
\end{aligned}
$$

subject to

$$
\begin{aligned}
\int r(1+i)^{t} z_{t j}^{*}\left(x_{t j}\right) \mathrm{d} x_{t j} & =1, \quad r(1+i)^{t} z_{t j}^{*}\left(x_{t j}\right) \geq 0 \forall t j, \\
\sum_{t=0}^{T} r \int x_{t j} z_{t j}^{*}\left(x_{t j}\right) \mathrm{d} x_{t j} & \leq C_{j}\left(1+\gamma^{*}\right)+\alpha_{j}^{*}, \\
\gamma^{*} & \geq 0, \quad \alpha_{j}^{*} \geq 0, \quad \forall j .
\end{aligned}
$$

At optimality:

$$
r(1+i)^{t} z_{t j}^{*}\left(x_{t j}\right)=\frac{f_{t j}\left(x_{t j}\right) \exp \left(-r s_{j} x_{t j}\right)}{\int f_{t j}\left(x_{t j}\right) \exp \left(-r s_{j} x_{t j}\right) \mathrm{d} x_{t j}}, \quad \forall t j .
$$

The dual program becomes more understandable with the following transformation of variables:

$$
y_{t j}^{*}\left(x_{t j}\right)=r(1+i)^{t} z_{t j}^{*}\left(x_{t j}\right), \quad \forall t j .
$$

The transformed dual is:

$$
\begin{aligned}
& \min \sum_{t=0}^{T} \frac{(1+i)^{-t}}{r} \sum_{j=1}^{n} \int y_{t j}^{*}\left(x_{t j}\right) \ln \left[y_{t j}^{*}\left(x_{t j}\right) / f_{t j}\left(x_{t j}\right)\right] \mathrm{d} x_{t j} \\
& +\sum_{j=1}^{n} \alpha_{j}^{*}+C \gamma^{*}
\end{aligned}
$$

subject to

$$
\begin{aligned}
\int y_{t j}^{*}\left(x_{t j}\right) \mathrm{d} x_{t j} & =1, \quad y_{t j}^{*}\left(x_{t j}\right) \geq 0, \quad \forall t j \\
\sum_{t=0}^{T}(1+i)^{-t} \int x_{t j} y_{t j}^{*}\left(x_{t j}\right) \mathrm{d} x_{t j} & \leq C_{j}\left(1+\gamma^{*}\right)+\alpha_{j}^{*}, \\
\gamma^{*} & \geq 0, \quad \alpha_{j}^{*} \geq 0, \quad \forall j .
\end{aligned}
$$

At optimality:

$$
\begin{aligned}
& \sum_{t=0}^{T}(1+i)^{-t} / r \sum_{j=1}^{n}\left(\ln \left(E\left[\exp \left(-r s_{j} x_{t j}\right]\right)\right)+\sum_{j=1}^{n} s_{j} C_{j}\right. \\
& \quad+\sum_{t=0}^{T}(1+i)^{-t} / r \sum_{j=1}^{n} \int y_{t j}^{*}\left(x_{t j}\right) \ln \left(y_{t j}^{*}\left(x_{t j}\right) / f_{t j}\left(x_{t j}\right)\right) \mathrm{d} x_{t j} \\
& \quad+\sum_{j=1}^{n} \alpha_{j}^{*}+C \gamma^{*}=0, \\
& y_{t j}^{*}\left(x_{t j}\right)=\frac{f_{t j}\left(x_{t j}\right) \exp \left(-r s_{j} x_{t j}\right)}{\int f_{t j}\left(x_{t j}\right) \exp \left(-r s_{j} x_{t j}\right) \mathrm{d} x_{t j}}, \quad \forall t j .
\end{aligned}
$$

In the dual, the objective is the present value of the entropy plus shadow costs associated with the budget constraint and the limitations on investments in projects of Equation (61). At optimality, this is equal to the profit described by Equation (65). $y_{t j}^{*}\left(x_{t j}\right)$ is a probability density function which is the original distribution, $f_{t j}\left(x_{t j}\right)$, discounted by the risky returns described by Equation (66). The constraint associated with $s_{j}$ equates to the present value of the returns to costs using the probability function of Equation (63).

We can see more about risky portfolios if the distribution is specified. Let us use the normal distribution.

\subsubsection{The normal case}

$$
\begin{aligned}
\min & {\left[\sum_{j=1}^{n} s_{j}^{2} \sum_{t=0}^{T} r(1+i)^{-t} \sigma_{t j}^{2} / 2\right.} \\
& \left.+\sum_{j=1}^{n} s_{j}\left(C_{j}-\sum_{t=0}^{T}(1+i)^{-t} \mu_{t j}\right)\right],
\end{aligned}
$$

subject to

$$
\begin{aligned}
\sum_{j=1}^{n} s_{j} C_{j} & \leq C, \\
0 & \leq s_{j} \leq 1, \quad \forall j .
\end{aligned}
$$

Dual program:

$$
\min \sum_{j=1}^{n} \frac{\left(s_{j}-C_{j}+\sum_{t=0}^{T}(1+i)^{-t} \mu_{t j}\right)^{2}}{2 r \sum_{t=0}^{T}(1+i)^{-t} \sigma_{t j}^{2}}+\sum_{j=1}^{n} \alpha_{j}^{*}+C \gamma^{*},
$$

subject to

$$
\begin{aligned}
& C_{j} \gamma^{*}+\alpha_{j}^{*}+s_{j}^{*} \geq 0, \quad \forall j, \\
& \gamma^{*} \geq 0, \quad \alpha_{j}^{*} \geq 0, \quad \forall j .
\end{aligned}
$$

The dual holds all the present value calculations and the normal random variables in a sum of quadratic terms in the $s_{j}^{*} . s_{j}^{*}$ is compared with the expected present value of the project $j$ and scaled by the variance of the project times the risk parameter $r$ in the objective described by Equation (70). This is the only term where $r$ appears and shows the 
close relationship between risk and variance. $s_{j}^{*}$ is related to the shadow prices in the constraints of Equation (71).

\subsection{The dynamic model with stochastic constraints}

For stochastic constraints, let us first convert the problem into a convex minimization program:

$\min \sum_{t=0}^{T}(1+i)^{-t} \sum_{j=1}^{n}\left(1 / r \ln \left(E\left[\exp \left(-r s_{j} x_{t j}\right)\right]\right)\right)+\sum_{j=1}^{n} s_{j} C_{j}$,

subject to

$$
\begin{aligned}
& \int \mathbf{1}_{N}\left[\sum_{t=0}^{T^{\prime}}(1+i)^{T^{\prime}-t} \sum_{j=1}^{n} s_{j} x_{t j}+(1+i)^{T^{\prime}}\left(C-\sum_{j^{\prime}=1}^{n} s_{j^{\prime}} C_{j^{\prime}}\right)\right] \\
& \quad \times \mathrm{d} F(x) \leq p_{T^{\prime}}, \quad 0 \leq T^{\prime} \leq T, \\
& \sum_{j=1}^{n} s_{j} C_{j} \leq C, \\
& 0 \leq s_{j} \leq 1, \quad \forall j,
\end{aligned}
$$

Dual program:

$$
\begin{aligned}
\min & \sum_{t=0}^{T} \sum_{j=1}^{n} \int z_{t j}^{*}\left(x_{t j}\right) \ln \left[z_{t j}^{*}\left(x_{t j}\right) r(1+i)^{t} / f_{t j}\left(x_{t j}\right)\right] \mathrm{d} x_{t j} \\
& +\sum_{j=1}^{n} \alpha_{j}^{*}+C \gamma^{*}+\sum_{T^{\prime}=0}^{T}(1+i)^{T^{\prime}} C q_{T^{\prime}}^{*}
\end{aligned}
$$

subject to

$$
\begin{aligned}
& \int w_{T^{\prime}}^{*}(x) \mathrm{d} F(x)+q_{T^{\prime}}^{*}=0, \quad \forall T^{\prime}, \\
& \int \mathbf{1}_{N}\left(-w_{T^{\prime}}^{*}(x)\right) \mathrm{d} F(x) \leq p_{T^{\prime}}, \quad \forall T^{\prime}, \\
& \int r(1+i)^{t} z_{t j}^{*}\left(x_{t j}\right) \mathrm{d} x_{t j}=1, \quad r(1+i)^{t} z_{t j}^{*}\left(x_{t j}\right) \geq 0 \quad \forall t j, \\
& \sum_{t=0}^{T} r \int x_{t j} z_{t j}^{*}\left(x_{t j}\right) \mathrm{d} x_{t j} \\
& \leq \sum_{T^{\prime}=0}^{T} \int\left(\sum_{t=0}^{T^{\prime}}(1+i)^{T^{\prime}-t} x_{t j}-(1+i)^{T^{\prime}} C_{j}\right) w_{T^{\prime}}^{*}(x) \mathrm{d} F(x) \\
& \quad+C_{j}\left(1+\gamma^{*}\right)+\alpha_{j}^{*}, \\
& \gamma^{*} \geq 0, \quad \alpha_{j}^{*} \geq 0, \quad \forall j .
\end{aligned}
$$

At optimality:

$$
r(1+i)^{t} z_{t j}^{*}\left(x_{t j}\right)=\frac{f_{t j}\left(x_{t j}\right) \exp \left(-r s_{j} x_{t j}\right)}{\int f_{t j}\left(x_{t j}\right) \exp \left(-r s_{j} x_{t j}\right) \mathrm{d} x_{t j}}, \quad \forall t j .
$$

The dual program becomes more understandable with the following transformation of variables:

$$
y_{t j}^{*}\left(x_{t j}\right)=r(1+i)^{t} z_{t j}^{*}\left(x_{t j}\right), \quad \forall t j .
$$

The transformed dual is:

$$
\begin{aligned}
\min & \sum_{t=0}^{T} \frac{(1+i)^{-t}}{r} \sum_{j=1}^{n} \int y_{t j}^{*}\left(x_{t j}\right) \ln \left[y_{t j}^{*}\left(x_{t j}\right) / f_{t j}\left(x_{t j}\right)\right] \mathrm{d} x_{t j} \\
+ & \sum_{j=1}^{n} \alpha_{j}^{*}+C \gamma^{*}-\sum_{T^{\prime}=0}^{T}(1+i)^{T^{\prime}} C \int w_{T^{\prime}}^{*}(x) \mathrm{d} F(x),
\end{aligned}
$$

subject to

$$
\begin{gathered}
\int \mathbf{1}_{N}\left(-w_{T^{\prime}}^{*}(x)\right) \mathrm{d} F(x) \leq p_{T^{\prime}}, \quad \forall T^{\prime}, \\
\int y_{t j}^{*}\left(x_{t j}\right) \mathrm{d} x_{t j}=1, \quad y_{t j}^{*}\left(x_{t j}\right) \geq 0, \quad \forall t j, \\
\sum_{t=0}^{T}(1+i)^{-t} \int x_{t j} y_{t j}^{*}\left(x_{t j}\right) \mathrm{d} x_{t j} \\
\leq \sum_{T^{\prime}=0}^{T} \int\left(\sum_{t=0}^{T^{\prime}}(1+i)^{T^{\prime}-t} x_{t j}-(1+i)^{T^{\prime}} C_{j}\right) w_{T^{\prime}}^{*}(x) \mathrm{d} F(x) \\
+C_{j}\left(1+\gamma^{*}\right)+\alpha_{j}^{*}, \quad \forall j, \\
\gamma^{*} \geq 0, \quad \alpha_{j}^{*} \geq 0, \quad \forall j .
\end{gathered}
$$

At optimality:

$$
y_{t j}^{*}\left(x_{t j}\right)=\frac{f_{t j}\left(x_{t j}\right) \exp \left(-r s_{j} x_{t j}\right)}{\int f_{t j}\left(x_{t j}\right) \exp \left(-r s_{j} x_{t j}\right) \mathrm{d} x_{t j}}, \quad \forall t j .
$$

For the case with stochastic constraints, we see many of the same properties as before. In addition, the stochastic constraints also appear in the dual of Equation (86), making the dual more complex than the primal. Let us see the result when the distribution is specified to be normal.

\subsubsection{The normal case}

$$
\begin{aligned}
& \min \left[\sum_{j=1}^{n} s_{j}^{2} \sum_{t=0}^{T} r(1+i)^{-t} \sigma_{t j}^{2} / 2\right. \\
& \left.+\sum_{j=1}^{n} s_{j}\left(C_{j}-\sum_{t=0}^{T}(1+i)^{-t} \mu_{t j}\right)\right],
\end{aligned}
$$

subject to

$$
\begin{aligned}
& \sum_{j=1}^{n} s_{j}\left(\sum_{t=0}^{T^{\prime}}(1+i)^{T^{\prime}-t} \mu_{t j}-(1+i)^{T^{\prime}} C_{j}\right)+(1+i)^{T^{\prime}} C \\
& \quad+z_{p_{T^{\prime}}} \sqrt{\sum_{j=1}^{n} s_{j}^{2}\left(\sum_{t=0}^{T^{\prime}}(1+i)^{2\left(T^{\prime}-t\right)} \sigma_{t j}^{2}\right)} \geq 0, \quad \forall T^{\prime}, \\
& \sum_{j=1}^{n} s_{j} C_{j} \leq C, \\
& 0 \leq s_{j} \leq 1, \quad \forall j .
\end{aligned}
$$

In the probability constraints of Equation (92), $z_{p_{T^{\prime}}}$ is the value of the standard normal for which the cumulative probability is $p_{T^{\prime}}$. For a cumulative probability of 0.05 that 
value would be equal to -1.645 . For negative values of $z_{p_{T^{\prime}}}$, the program is convex and has a dual, which is given below.

\section{Dual program}

$$
\begin{gathered}
\min \sum_{j=1}^{n} \frac{\left(s_{j}^{*}-C_{j}+\sum_{t=0}^{T}(1+i)^{-t} \mu_{t j}\right)^{2}}{2 r \sum_{t=0}^{T}(1+i)^{-t} \sigma_{t j}^{2}} \\
+\sum_{j=1}^{n} \alpha_{j}^{*}+C \gamma^{*}+\sum_{T^{\prime}=0}^{T}(1+i)^{T^{\prime}} C \nu_{T}^{*},
\end{gathered}
$$

subject to

$$
\begin{aligned}
& \sum_{j=1}^{n}\left(w_{T^{\prime}}^{*}\right)^{2} \leq z_{p_{T^{\prime}}}^{2}, \quad \forall T^{\prime}, \\
& \sum_{T^{\prime}=0}^{T}\left[\frac{w_{T^{\prime} j}^{*}}{\left(\sum_{t=0}^{T^{\prime}}(1+i)^{2\left(T^{\prime}-t\right)} \sigma_{t j}^{2}\right)}\right. \\
& \left.\quad-\left(\sum_{t=0}^{T^{\prime}}(1+i)^{T^{\prime}-t} \mu_{t j}-(1+i)^{T^{\prime}} C_{j}\right) v_{T^{\prime}}^{*}\right] \\
& \quad+C_{j} \gamma^{*}+\alpha_{j}^{*}+s_{j}^{*} \geq 0, \quad \forall j, \quad{ }^{\prime}, \quad w_{T^{\prime} j} \geq 0, \quad \forall T^{\prime}, \quad j .
\end{aligned}
$$

The stochastic constraints in the primal add quadratic constraints to the dual of the dynamic portfolio problem described by Equation (96). The $w_{T^{\prime} j}^{*}$, are the contribution to the value squared of $z_{p_{T^{\prime}}}$, from the project $j$ in time pe$\operatorname{riod} T^{\prime} . v_{T^{\prime}}^{*}$ is the dual variable for the stochastic constraint on the accounts at time period $T^{\prime}$ given by Equation (92). All in all, the dual is a quadratically-constrained quadratic program, which is a reasonable way to solve the dynamic portfolio problem with stochastic constraints. See Peterson and Ecker (1970) for more analysis of this class of programs.

\section{Results and conclusions}

In this paper we have analyzed risky portfolio problems, both non-parametric and parametric, in static, dynamic, and stochastic versions. All were found to be convex and amenable to the calculation of a dual. For the nonparametric case, the dual was found by embedding the problem in function space and using functional conjugate duality. For the parametric (normal) case, the generalized geometric programming form of conjugate duality was used. This analysis leads to insights into risky portfolios, which are useful to both practitioners and to problem solvers.

The static model provides a linkage between certainty equivalents and constrained entropy. For the normal case, a recommended value of the risk parameter is related to the reciprocal of the portfolio standard deviation.
The dynamic model transforms the present value of the certainty equivalent into the present value of the entropy over a distribution, which is discounted by the risk parameter for high returns. Thus, we see the two types of discounting (over time and over risk) working together.

Adding stochastic constraints to the dynamic model leads to a complicated but convex dual in the nonparametric case. In the normal case, the dual is a quadratically-constrained quadratic program, amenable to solution. Each stochastic constraint transforms to a simple quadratic constraint in the dual.

Overall there is a close relationship among risk, variance, certainty equivalents, moment generating functions and entropy. There is also a link between discounting over time with interest and over return with risk. This analysis should encourage the use and understanding of these concepts in managing uncertainty and risk.

\section{References}

Birge, J.R. and Louveaux, F. (1997) Introduction to Stochastic Programming, Springer, New York, NY.

Duffin, R.J., Peterson, E.L. and Zener, C. (1967) Geometric Programming - Theory and Application, Wiley, New York, NY.

Fang, S.-C., Rajasekera, J.R. and Tsao, H.-S. J. (1997) Entropy Optimization and Mathematical Programming, Kluwer, Boston, MA.

Jefferson, T.R., Scott, C.H. and Cozzolino, J.M. (1979) The analysis of risky portfolios by geometric programming. Zeitschrift für Operations Research, 23, 207-217.

Jorion, P. (2000) Value at Risk: A New Benchmark for Measuring Financial Risk, McGraw-Hill, New York.

Kall, P. and Wallace, S.W. (1994) Stochastic Programming, Wiley, Chichester, UK.

Markowitz, H.M. (1952) Portfolio selection. Journal of Finance, 7, 77-91. Merton, R. (1990) Continuous Time Finance, Basil Blackwell, Oxford, UK.

Peterson, E.L. (1976) Geometric programming. SIAM Review, 18, 1-52. Peterson, E.L. and Ecker, E.G. (1970) Geometric programming: duality in quadratic programming and $l_{p}$-approximation, in Proceedings of the Princeton Symposium on Mathematical Programming, Princeton University Press, Princeton, NJ, pp. 445-480.

Pratt, J.W. (1964) Risk aversion in the small and in the large. Econometrica, 32, 122-136.

Prekopa, A. (1995) Stochastic Programming, Springer-Verlag, New York. Rockafellar, R.T. and Uryasev, S. (2000) Optimization of conditional value-at-risk. The Journal of Risk, 2, 21-41.

Scott, C.H. and Jefferson, T.R. (1977) Duality in infinite dimensional mathematical programming: convex integral functionals. Journal of Mathematical Analysis and Applications, 61, 251-261.

Uryasev, S.P. (ed.) (2000) Probabilistic Constrained Optimization: Methodology and Applications, Kluwer, Dordrecht, The Netherlands.

Ziemba, W.T. and Mulvey, J.T. (1998) Worldwide Asset and Liability Modeling, Cambridge University Press, Cambridge, UK.

\section{Biographies}

Thomas R. Jefferson is a Visiting Professor of Management in the Faculty of Business Administration, Bilkent University, Ankara, Turkey. He holds a Ph.D. in Management Science from Northwestern University, and an M.Sc. in Statistics and a B.Sc. in Mathematics from the University of Toronto. He has also held academic positions at such universities as Montreal, New South Wales, Pittsburgh, Tulane, and 
California (Los Angeles). His research focus is in optimization with particular interest in the modeling, analysis and solution of nonlinear programs. He is presently writing a research monograph on geometric programming with Carlton H. Scott.

Carlton H. Scott is a Professor in the Graduate School of Management at the University of California, Irvine. He has a Ph.D. in Theoretical Physics from the University of New South Wales, Australia and subsequently worked as an Operations Research Analyst in the steel industry. Prior to joining UCI, he was on the faculty of the School of Mechanical and Industrial Engineering at University of New South Wales for 10 Years. His research is centered around nonlinear mathematical programming where he is widely published. His contributions have spanned theory, applications and algorithms. 